\title{
INTERCÂMBIO ACADÊMICO INTERNACIONAL EM ODONTOLOGIA: UM RELATO DE EXPERIÊNCIA
}

\author{
INTERNATIONAL ACADEMIC MOBILITY IN TRAINING IN DENTISTRY: AN \\ EXPERIENCE REPORT
}

\author{
Lisia Daltro Borges Alves, Hervânia Santana da Costa
}

Universidade Estadual de Feira de Santana - UEFS

\begin{abstract}
The objective of this study was to report the experiences and activities developed by a student of Dentistry of the State University of Feira de Santana, through the international academic mobility program, at Universidad del País Vasco, Bilbao - Spain, from August 2015 to July 2016. This is a descriptive study of the experience report type. On this occasion, it was possible to perform Spanish language improvement, which gave a greater mastery of the foreign language, attending theoretical and practical classes of Dentistry of the academic program of the Universidad del País Vasco, allowing contact with new didactics and technologies. In addition, the student was able to participate and present papers in scientific events, enabling contact with international researchers. An internship was also held at the University Hospital of Cruces, where he observed and experienced the operation of the Vasco Public Health System. The exchange and these activities offered opportunities for the personal, professional and technical-scientific improvement of the student.
\end{abstract}

Key words: International Education Exchange; Dentistry; Higher Education

\section{Resumo}

O objetivo deste estudo foi relatar as experiências e atividades desenvolvidas por uma discente de Odontologia da Universidade Estadual de Feira de Santana, por meio do programa de mobilidade acadêmica internacional, na Universidad del País Vasco, Bilbao - Espanha, no período de agosto de 2015 à julho de 2016. Trata-se de um estudo descritivo, do tipo relato de experiência. Nesta oportunidade, foi possível realizar aperfeiçoamento linguístico do espanhol, o que conferiu um maior domínio da língua estrangeira, frequentar aulas teóricas e práticas de Odontologia do programa acadêmico da Universidade del País Vasco, permitindo contato com novas didáticas e tecnologias. Além disso, a discente pôde participar e apresentar trabalhos em eventos científicos, possibilitando o contato com pesquisadores internacionais. Realizou-se ainda estágio no Hospital Universitário de Cruces, onde observou e vivênciou o funcionamento do Sistema Público de Saúde Vasco. A realização do intercâmbio e destas atividades ofereceram oportunidades para o aperfeiçoamento pessoal, profissional e técnico-científico da discente.

Palavras chave: Intercâmbio Educacional Internacional; Odontologia; Educação Superior 


\section{Introdução}

A realização do intercâmbio acadêmico é uma experiência única e um sonho para muitos estudantes. Com a globalização, cada vez faz-se mais importante que a formação dos profissionais seja composta de conhecimentos e aptidões diversas e capazes de acompanhar o desenvolvimento tecnológico ${ }^{1}$.

$\mathrm{O}$ intercâmbio acadêmico confere inúmeros benefícios àquele que o realiza. No contexto da formação acadêmica/profissional, permite-lhe a vivência com novos professores, com metodologias e rotinas distintas; conhecimento de estudos e pesquisadores internacionais; contato com novas tecnologias e a utilização de diferentes técnicas na odontologia².

Esta experiência confere vantagens que vão além do aprendizado técnico, uma vez que contribuem também para o desenvolvimento psicológico, amadurecimento, autoconfiança, desenvolvimento psicológico e independência do indivíduo ${ }^{3}$. Viver em outro país permite o conhecimento de hábitos e culturas, o aperfeiçoamento de uma língua estrangeira, além de auxiliar na superação de dificuldades e na capacidade de adaptação ${ }^{4,5}$.

Nesse sentido, foi criado o Ciências sem Fronteiras (CSF), que é um programa que visa permitir o contato de estudantes brasileiros com a ciência, a tecnologia e a inovação de diversas partes do mundo, utilizando o intercâmbio e a mobilidade internacional. Um dos principais objetivos deste programa é a pesquisa científica, pois busca criar e manter vínculos entre os estudiosos dos outros países com os pesquisadores do Brasil, assim como, atrair estes estudiosos para pesquisar em nosso país. 0 subsídio deste programa prevê a isenção das taxas acadêmicas no espaço europeu e concede bolsas referentes a gastos com moradia, materiais didáticos, seguro saúde, passagens aéreas e um valor fixo mensal para as demais despesas $^{6}$.

O objetivo do presente artigo foi relatar as atividades desenvolvidas e descrever as experiências vivenciadas por uma discente de Odontologia da Universidade Estadual de Feira de Santana (UEFS), em programa de mobilidade acadêmica internacional, na Universidad del País Vasco, Bilbao (UPV) - Espanha, de agosto de 2015 à julho de 2016.

\section{Metodologia}

Consiste de um estudo descritivo, do tipo relato de experiência a respeito da vivência de uma discente de Odontologia da Universidade Estadual de Feira de Santana (UEFS) em programa de mobilidade acadêmica internacional, na Universidad del País Vasco, Bilbao (UPV) Espanha, de agosto de 2015 à julho de 2016. Foram descritos quatro grupos de atividades que foram desenvolvidas pela discente durante o intercâmbio: curso de espanhol, programa acadêmico, iniciação científica e estágio de verão. O curso de espanhol foi organizado pela UPV e realizado em agosto de 2015. O programa acadêmico seguiu o período letivo da UPV, de setembro de 2015 a junho de 2016, contando com atividades que em sua maioria foram desenvolvidas no campus da UPV e em algumas disciplinas específicas, no Hospital Universitário de Cruces (HUC). As atividades de iniciação científica ocorreram de forma concomitante ao programa acadêmico enquanto que, ao final do intercâmbio, de junho a agosto de 2016, foi realizado o estágio de verão, com atividades exclusivamente desenvolvidas no HUC.

\section{Resultados e Discussão}

\section{Curso de Espanhol}

Apesar de ser exigido pelo CSF um nível de proficiência em espanhol, com níveis mínimos $A 2$ ou B1 certificados através do DELE, foi realizado um curso de férias com todos os intercambistas que ingressariam na UPV em setembro de 2015. Para o curso foi disponibilizado material didático em formato de livro, ministradas aulas teóricas, apresentações de trabalhos orais, realizadas provas escritas e uma aula de campo em pontos turísticos de Bilbao.

Este curso permitiu o aperfeiçoamento da língua estrangeira em um momento fundamental, deixando a aluna mais preparada tanto para as aulas do programa acadêmico que se iniciariam, como para a vivência no novo país de residência.

\section{Programa Acadêmico}

O programa acadêmico foi realizado junto ao curso de Graduação em Odontologia da UPV, entre setembro de 2015 e junho de 2016. A discente cursou oito disciplinas ministradas em espanhol/castelhano durante dois quadrimestres.

O sistema utilizado pela Universidad del País Vasco incluía aulas teóricas ministradas pelos professores, apresentação de trabalhos orais pelos alunos, provas escritas e atividades práticas que variavam de acordo com a disciplina. Nas disciplinas Patología y Terapéutica Dentales II, Dolor Orofacial, Cirugía II e Farmacología Aplicada y Urgencias en Odontología as práticas 
ocorriam a nível ambulatorial nas clínicas da UPV com o atendimento de pacientes. Em Gestión y Ejercício de la Profesión Odontológica eram realizadas práticas sem pacientes, nas quais eram analisadas se as clínicas cumpriam as normativas estabelecidas pela vigilância sanitária, discutia-se o território e o mercado, simulando a abertura de clínicas odontológicas, calculava-se gastos fixos e variáveis de um consultório, e simulava-se entrevistas de emprego. As disciplinas que envolviam a temática cirurgia (Cirugía Maxilofacial e Cirugía II) incluíam também práticas no Hospital Universitário de Cruces, onde eram acompanhados os atendimentos do cirurgião bucomaxilofacial e depois eram redigidos relatórios.

A respeito da estrutura, a UPV contava com dez ambulatórios, cada um com 6 cadeiras odontológicas, 1 aparelho para tomada radiográfica digital, 2 computadores e 1 aparelho para revelação das radiografias digitais. Ademais, a UPV conta com um laboratório para a realização de radiografias panorâmicas e outro de prótese, que durante as aulas práticas, os técnicos especializados ficavam disponíveis em ambos os laboratórios, para a realização de radiografias e trabalhos protéticos.

A recepção da UPV desempenhava diversas funções, como a organização e disponibilização dos prontuários impressos, gerenciamento, transferência e agendamento dos pacientes e recebimento dos pagamentos referentes aos tratamentos realizados.

Os prontuários clínicos dos pacientes eram compostos por uma parte impressa (orçamento, termo de consentimento livre esclarecido, ficha com odontograma, cronograma de atendimento) e outra digital. O sistema utilizado era o Aladium, onde alunos e professores possuíam login e senha para o acesso. Neste sistema eram reveladas as radiografias digitais por meio de um dispositivo específico e anexadas aos respectivos prontuários digitais dos pacientes. Um filme radiográfico foi cedido pela universidade à cada aluno, sob o pagamento de uma fiança, ressarcida no momento da devolução do material ao final do período do intercâmbio. Durante a tomada radiográfica, o filme era revestido por uma capa plástica descartável que permitia a sua reutilização.

Além disso, cada aluno possuía uma agenda digital. Nela, o aluno informava o código do paciente no dia e horário que iria atendê-lo para que a recepção o agendasse e tivesse conhecimento prévio de quais prontuários deveriam ser disponibilizados nas clínicas. Nos casos em que o paciente avisasse à recepção que não poderia comparecer, o funcionário da recepção enviava uma mensagem de texto para o número do aluno informando o fato. Diante da necessidade de um paciente novo, digitava-se o código 8 no dia e horário, especificando a disciplina e assim, a recepção agendava um paciente previamente triado ou que tivesse sido transferido de outra disciplina. Os tratamentos só eram iniciados após o paciente receber, concordar e assinar o orçamento, confeccionado pelo aluno, tendo como base a lista de preços estabelecida pela universidade. Desta forma, o paciente pagava cada procedimento após sua realização.

A respeito da paramentação, na UPV utilizava-se apenas o jaleco por cima da roupa, sem padronização (podendo o aluno estar vestido com saias e calçando sapatos abertos) ou um pijama branco padrão da universidade que só era exigido nas disciplinas de cirurgia. Eram utilizadas luvas e máscara, entretanto, não era obrigatório o uso de gorros, sobre luvas e óculos de proteção.

No que se refere à disponibilização de materiais e equipamentos, na UPV, os materiais de consumo (luva, guardanapo, babador, sugador, moldeiras e bandejas plásticas) ficavam disponíveis nas bancadas da clínica. Os demais materiais como anestésico, resina, material de moldagem, fotopolimerizador, kit de polimento de restauração ou turbinas deveriam ser solicitados no armazém, onde a retirada e a devolução eram registradas no sistema através de um cartão com código de barras que identificava cada aluno.

Quanto aos instrumentais, cada aluno recebeu duas chaves referentes à dois armários numerados igualmente, porém localizados em salas diferentes. Em um, ficavam disponíveis os materiais estéreis; no outro, eram colocados os materiais utilizados, sendo os profissionais da central de esterilização, os responsáveis pela desinfecção, esterilização e recolocação dos mesmos no armário de materiais estéreis. Além disso, havia uma autoclave pequena nas clínicas, onde as turbinas eram esterilizadas pelos alunos.

No que se refere à tutoria, para cada clínica era designado um professor tutor que orientava 6 duplas de alunos desde o planejamento do procedimento até a execução englobando os procedimentos das especialidades odontológicas contempladas na disciplina.

\section{Iniciação Científica}

Antes da realização do intercâmbio, a discente integrava o Núcleo de Câncer Oral 
(NUCAO) na UEFS, por esta razão, a discente buscou professores em diferentes áreas na UPV para a realização de pesquisa durante o ano de mobilidade acadêmica. Entretanto, a pesquisa na UPV, apesar de existente e de qualidade, era realizada quase que exclusivamente pelos professores. Existiam grupos de pesquisa, entretanto, os alunos que participavam eram prioritariamente integrantes dos cursos de pós graduação e o incentivo à pesquisa para alunos de graduação era muito pequeno.

Apesar de não conseguir se inserir- em um grupo de pesquisa, nem participar de um projeto durante o intercâmbio, a discente participou de congressos e simpósios onde apresentou trabalhos científicos desenvolvidos no NUCAOUEFS. Desta forma o intercâmbio foi também uma oportunidade para a divulgação de pesquisas produzidas na universidade de origem e escutar sugestões/críticas para aperfeiçoamento dos estudos.

\section{Práticas de verão}

A última etapa do programa foram as práticas de verão, momento em que a discente realizou um estágio observacional no setor de Cirurgia Bucomaxilofacial do Hospital Universitário de Cruces, sob a tutoria do Dr. Joseba Andoni Santamaría Zuazua, de junho à agosto de 2016.

Os atendimentos do tutor no Hospital de Cruces diferiam a depender do dia da semana. Um dos dias era destinado às consultas, enquanto que o outro era reservado para os procedimentos cirúrgicos. As consultas podiam ser de três tipos: 1a consulta, retorno após a realização dos exames e acompanhamento póscirúrgico.

Nas consultas de 1a vez, os procedimentos de anamnese (registro das queixas do paciente e da história da doença) e de exame físico extra e intraorais eram realizados. Após suspeita diagnóstica, diante da indicação de tratamento cirúrgico, os exames complementares eram solicitados. Nas consultas de retorno, os resultados dos exames solicitados eram analisados e quando estes não apresentavam alterações, eram fornecidas as recomendações pré-operatórias. Em casos de cirurgias orais menores, o agendamento da cirurgia era autorizado na recepção. Em casos de pacientes diagnosticados com lesões malignas, o cirurgião Bucomaxilofacial realizava o agendamento e o procedimento deveria ocorrer em um prazo máximo de 30 dias obedecendo uma política do Hospital de Cruces e do setor de Cirurgia
Maxilofacial.

O terceiro tipo de consulta era a de acompanhamento. Todos os pacientes que haviam sido submetidos à cirurgia reparadora (queiloplastias, palatoplastias) e, sobretudo, ressectivas de lesões malignas tinham um acompanhamento constante. A frequência destas consultas variava de acordo com o tipo de cirurgia realizada e o tempo de recuperação do paciente.

Nos dias de procedimento cirúrgico, o intervalo entre os pacientes variava de 15 a 30 minutos considerando que este momento era exclusivamente para o procedimento cirúrgico propriamente dito. As cirurgias mais frequentes eram exodontias de terceiros molares e biópsias de lesões em tecidos moles.

Uma característica que mereceu destaque é o sistema de informação digital utilizado pelo Hospital Universitário de Cruces. Cada médico possuía um login, por meio do qual conseguia visualizar agenda, prontuário digital do paciente, registrar informações sobre as consultas, tecer comentários, solicitar e visualizar os resultados dos exames.

A participação de estudantes de graduação em programas de mobilidade acadêmica internacional é uma oportunidade única para o crescimento pessoal e profissional daquele que o vive. Morar durante um ano em Bilbao - Espanha, e estar vinculada à Universidad del País Vasco, permitiu que a discente em questão aperfeiçoasse o domínio da língua espanhola, cursasse disciplinas do programa acadêmico desta instituição, participasse e apresentasse trabalhos em congressos científicos à nivel internacional e realizasse estágio em um hospital do Sistema Público de Saúde do País Vasco.

Dentre as muitas contribuições que o intercâmbio oferece ao estudante que o realiza, está o aperfeiçoamento de uma língua estrangeira. Ao encontrar-se imerso em um novo país, realizando atividades sociais e acadêmicas diariamente, a fluência e o domínio da nova língua são facilitados por este processo. Apesar da exigência de um nível de proficiência mínimo, os cursos de língua estrangeira oferecidos no início do intercâmbio são essenciais para auxiliar neste processo de adaptação. Assim como relatado por este estudo, Guskuma et al. ${ }^{5}$, também recebeu curso preparatório no início da sua estância na Irlanda 5 .

A língua estrangeira representa um fator tão importante neste contexto de mobilidade acadêmica, que Dalmolin et al. ${ }^{7}$ relatou que suscitou dúvidas a respeito do intercâmbio por realizá-lo em Portugal e consequentemente, não 
estar desenvolvendo habilidade com um novo idioma ${ }^{7}$. Neste contexto, o estudo de Pithan et al. ${ }^{8}$ analisou os depoimentos de 13 alunos de graduação em odontologia que realizaram intercâmbio acadêmico, e observou que o contato com a língua estrangeira para alguns alunos foi considerado como fator motivacional, sendo inclusive em muitos casos, fator determinante na escolha do país ${ }^{8}$. Ademais, para outros autores, a falta do domínio do idioma resulta em dificuldades durante a realização do intercâmbio, tanto durante o processo de aprendizagem, por impactar diretamente na comunicação entre alunos e professores, como na interação social e na compreensão cultural ${ }^{8,9}$.

A participação no programa acadêmico de graduação durante o intercâmbio, como descrita nesse relato, destaca-se sobretudo por permitir uma análise crítica e comparação entre as metodologias, conteúdos e estruturas da universidade de origem e da instituição internacional, corroborando com o relatado por outros autores ${ }^{10}$. Além disso, o aluno tem a oportunidade de cursar disciplinas que podem não constar na grade curricular do seu curso de graduação no Brasil, conferindo à sua formação mais um fator diferencial ${ }^{5}$.

Especificamente para a graduação em odontologia, os atendimentos clínicos são fundamentais durante o processo de formação do novo profissional. Nesse contexto, o intercâmbio permite que o aluno tenha contato com novas técnicas, tecnologias e ideias no exterior, como relatado no presente estudo e por Dalmolin et $a l_{.}{ }^{7}$. Da mesma forma, Pithan et $a .^{8}$ destaca que este tipo de atividade atua positivamente tanto na relação com os pacientes e no enfrentamento de desafios, como na qualificação do profissional egresso para o trabalho de forma integrada à globalização intelectual $^{8}$. Entretanto, alguns autores como Silva et al., ${ }^{2}$ e Guskuma et al., ${ }^{5}$ relataram que durante o intercâmbio não puderam realizar atendimentos clínicos, sendo um dos fatores citados a barreira linguística ${ }^{2,5}$.

A possibilidade de inserção ou de desenvolver projetos de pesquisa durante o intercâmbio é uma oportunidade que agrega muito valor na formação do acadêmico que o realiza. No relato de Silva et al., ${ }^{2}$ este objetivo foi alcançado, e houve o desenvolvimento de pesquisa neste período, enquanto que Guskuma et al., ${ }^{5}$ e no presente relato, esta oportunidade não esteve presente ${ }^{2,5}$. Outra ponto de destaque é a chance de participar de eventos científicos internacionais, que neste contexto torna-se não só um meio de aquisição de conhecimento teórico e contato com grandes estudiosos, como um excelente cenário para a divulgação de pesquisas desenvolvidas nas instituições brasileira, assim como relatado neste estudo e no estudo de Dalmolin et al. ${ }^{7}$.

Ao realizar as práticas de verão no HUC, que integra o sistema público de saúde do País Vasco, foi possível compará-lo quanto aos seus aspectos físicos, disponibilidade de tecnologia, organizacionais, programas e políticas de saúde, com Sistema Único de Saúde do Brasil, fatores também destacados por outros autores ${ }^{5,7,9}$. Para Guskuma et al. ${ }^{5}$, notar, comparar e refletir sobre essas diferenças é de fundamental importância para futuras propostas de mudanças no país de origem $^{5}$. Ademais, Freitas et al. ${ }^{9}$ destaca que essas atividades contribuem positivamente para o pensamento crítico e uma maior valorização e respeito por sua própria cultura? .

Além disso, é importante destacar que a maioria dos relatos de experiência que versam sobre mobilidade acadêmica são na área da enfermagem, sejam de graduação ${ }^{3,5,7,9}$ ou pós graduação ${ }^{4}$. Deste modo, é essencial que os estudantes de odontologia sejam instigados a relatar suas experiências, para que novos estudantes sejam encorajados a realizar esta experiência tão enriquecedora.

Desta forma, a mobilidade acadêmica internacional é uma estratégia de grande relevância para crescimento da Odontologia e suas contribuições vão desde uma maior qualificação durante a formação profissional, à um olhar diferenciado nos serviços de saúde prestados à população. Destaca-se ainda que apesar da graduação apresentar uma grade curricular que direciona a formação dos alunos, cada graduando é ator principal da sua própria trajetória acadêmica e o seu diferencial será formado através do seu enfrentamento para a superação de dificuldades, e aproveitamento das oportunidades que lhe são oferecidas.

\section{Conclusão}

A realização de um intercâmbio acadêmico ofereceu oportunidades para o aperfeiçoamento pessoal, profissional e técnico-científico da discente. Possibilitou o conhecimento e contato com a cultura Vasca e incentivou o crescimento pessoal, a medida que lhe conferiu resiliência e independência. Além disso, oportunizou o aperfeiçoamento e domínio da língua espanhola.

A experiência internacional propiciou uma visão reflexiva e crítica acerca da estrutura, organização e metodologia empregadas na universidade espanhola. Permitiu o contato com novas tecnologias dentro da odontologia, a 
participação e apresentação de trabalhos em eventos científicos. Além de ter viabilizado um contato com o meio hospitalar do Serviço Público de Saúde Vasco, destaca-se a vivência no serviço por meio do conhecimento e acompanhamento do plano de diagnóstico e tratamento precoce do câncer oral.

Por fim, este relato de experiência deseja incitar o apoio por parte das instituições financiadoras e de ensino superior à mobilidade acadêmica internacional, bem como encorajar os estudantes a se permitirem viver essa experiência enriquecedora em todos os sentidos.

\section{Referência}

1. Santos BS. Pela mão de Alice: o social e o político na pós-modernidade. 13th ed. São Paulo: Cortez; 2010.

2. Silva FB da, Silva CHF da, Fiamengui Fllho JF, Carneiro SV. Graduação saduíche: relato de experiência de estudante de odontologia da unicatólica em universidades norte-americanas. Rev Expressão Católica. 2016;1(1):75-80.

3. Oliveira MG de, Pagliuca LMF. Programa de mobilidade acadêmica internacional em enfermagem: relato de experiência. Rev Gaúcha Enferm [Internet]. 2012;33(1):195-8. Available from:

https://seer.ufrgs.br/RevistaGauchadeEnfermage m/article/view/19288/17018

4. Souza KV de. Intercâmbio educacional internacional na modalidade doutorado sanduíche em enfermagem: relato de experiência. Esc Anna Nery [Internet]. 2008;12(2):358-63. Available from: http://dx.doi.org/10.1590/S141481452008000200025

5. Guskuma EM, Dullius AA dos S, Godinho MLS da C, Costa MST, Terra F de S. Mobilidade acadêmica internacional na formação em enfermagem: relato de experiência. Rev Bras Enferm [Internet]. 2016;69(5):986-90. Available from:

http://www.scielo.br/pdf/reben/v69n5/00347167-reben-69-05-0986.pdf

6. O programa - Ciência sem fronteiras. [Internet]. Brasil, Ministério da Educação, CNPq, CAPES. 2018 [cited 2018 Aug 11]. Available from: http://www.cienciasemfronteiras.gov.br/web/csf /o-programa

7. Dalmolin IS, Pereira ER, Silva RMCR a., Gouveia MJB, Sardinheiro JJ. Intercâmbio acadêmico cultural internacional: uma experiência de crescimento pessoal e científico. Rev Bras Enferm. 2013;66(3):442-7.

8. Pithan SA, Nunes MF, Pires LC. Ciência sem Fronteiras na formação profissional: percepções de estudantes de Odontologia da UFSM. Rev da ABENO [Internet]. 2018 Mar 28;18(1):2-14. Available from: https://revabeno.emnuvens.com.br/revabeno/ar ticle/view/402

9. Freitas G, Reis GT, Coelho S, Oliveira M De, Reis CC. Intercâmbio internacional nos cursos de graduação de enfermagem International. J Nurs Heal. 2017;7(2):2013-25.

10. Dias IMÁV, Santos R da S. Relato de experiência de intercâmbio Brasil/Canadá: conhecendo o programa interdisciplinar de intervenção familiar. Esc Anna Nery R Enferm. 2007;11(2):365-9.

\section{Endereço para Correspondência}

Universidade Estadual de Feira de Santana - UEFS

Av. Transnordestina, s/n - Novo Horizonte, Feira de Santana - BA

CEP.: 44036-900

e-mail: lisia_94@hotmail.com

Recebido em 30/11/2018

Aprovado em 10/09/2019

Publicado em 13/02/2020 